\title{
Structural and mechanical properties of organogels: Role of oil and gelator molecular structure
}

\author{
Miguel A. Cerqueira ${ }^{\mathrm{a}, *}$, Luiz H. Fasolin ${ }^{\mathrm{c}}$, Carolina S.F. Picone ${ }^{\mathrm{c}}$, Lorenzo M. Pastrana ${ }^{\mathrm{a}}$, \\ Rosiane L. Cunha ${ }^{c}$, António A. Vicente ${ }^{b}$ \\ a International Iberian Nanotechnology Laboratory, Av. Mestre José Veiga s/n, 4715-330 Braga, Portugal \\ b Centre of Biological Engineering, University of Minho, Campus de Gualtar, 4710-057 Braga, Portugal \\ c Department of Food Engineering, Faculty of Food Engineering, University of Campinas - UNICAMP, CEP: 13083-862 Campinas, SP, Brazil
}

\section{A R T I C L E I N F O}

\section{Article history:}

Received 28 October 2016

Received in revised form 7 March 2017

Accepted 10 March 2017

Available online 12 March 2017

\section{Keywords:}

Gels

Oleogels

Organogels

Organogelator

Crystallization

Surfactant

\begin{abstract}
A B S T R A C T
This work aims at evaluating the influence of oil and gelator structure on organogels' properties through rheological measurements, polarized microscopy and small-angle X-ray scattering (SAXS). Four different food-grade gelators (glyceryl tristearate - GT; sorbitan tristearate - ST; sorbitan monostearate - SM and glyceryl monostearate - GM) were tested in medium-chain triglyceride and high oleic sunflower (MCT and LCT, respectively) oil phases. Organogels were prepared by mixing the oil phase and gelator at different concentrations ( 5 , $10,15,20$ and $25 \%$ ) at $80^{\circ} \mathrm{C}$ during $30 \mathrm{~min}$. All organogels presented birefringence confirming the formation of a crystalline structure that changed with the increase of the gelator concentration. Through the evaluation of SAXS peaks it has been confirmed that all structures were organized as lamellas but with different $d$-spacing values. These particularities at micro- and nanoscale level lead to differences in rheological properties of organogels. Results showed that the oil type (i.e. medium- and long-chain triglyceride) and hydrophilic head of gelators (i.e. sorbitan versus glyceryl) exert influence on the organogels physical properties, but the presence of monostearate leads to the formation of stronger organogels. Moreover, gels produced with LCT were stronger and gelled at lower organogelator concentration than MCT.
\end{abstract}

@ 2017 Elsevier Ltd. All rights reserved.

\section{Introduction}

Edible organogels, or oleogels, have recently generated an enormous interest for scientific and technological reasons in several fields including cosmetic, pharmaceutic and food science. In foods, edible oleogels have also called the attention due to their unique properties that allow shaping foods in terms of e.g. texture and appearance, while also allowing the introduction of e.g. bioactive or functional compounds. Additionally, the emerging of organogels are related with: a) the thermoreversibility between solid and liquid by heating above and cooling below the transition temperature, which changes according to physicochemical properties of the gelator and the continuous phase (Abraham et al., 2012; Toro-Vazquez et al., 2013); and b) the potential health benefits that they may bring if used as alternative ingredient in foods containing saturated and/or trans fatty acids, as they have shown the capacity to provide texture to food products (Rogers, Wright, \& Marangoni, 2009).

\footnotetext{
* Corresponding author.

E-mail addresses: miguelribeirocerqueira@gmail.com, miguel.cerqueira@inl.int (M.A. Cerqueira).
}

Organogels can be formed by the self-assembly of surfactant molecules (organogelators) into three-dimensional networks that entrap an organic liquid through capillary forces (Terech \& Weiss, 1997). They can be produced due to physical forces and chemical interactions depending on the kind of gelator used: polymeric or low molecular weight organogelators (LMOGs) (Vintiloiu \& Leroux, 2008) with molecular weight lower than 3000 Da (Abdallah \& Weiss, 2000). Gelator structure exerts a great influence on organogel properties since the ability to form supramolecular gels is only possible with very specific materials. The gelling ability is related to the balance between the soluble and insoluble fractions of the gelator in the solvent. The molecule must be relatively insoluble to self-assemble into anisotropic structures. On the other hand, it must have a soluble fraction to interact with the oil moieties (Co \& Marangoni, 2012). Despite the recently published studies using different gelators on organogels development (Davidovich-Pinhas, Barbut, \& Marangoni, 2016; Martins, Cerqueira, Fasolin, Cunha, \& Vicente, 2016; Valoppi et al., 2017), it is still of great interest to explore how the chemical structure of the gelator influences organogel formation at molecular level. The relationship between the structures, their structural specific characteristics and rheological properties is of utmost interest aiming their use in 
technological applications. In addition, the oil phase properties (e.g. triglycerides' carbon chains size and degree of saturation) change the way in which gelators generate a three-dimensional network (Gravelle, Davidovich-Pinhas, Zetzl, Barbut, \& Marangoni, 2016) and must be studied. Recently, the development of organogels using food grade materials has been increasing due to their possible applications in food and pharmaceutical products. Organogels using ethyl cellulose (Davidovich-Pinhas, Barbut, \& Marangoni, 2015a; Gravelle et al., 2016), waxes (Martins et al., 2016; Toro-Vazquez, Alonzo-Macias, Dibildox-Alvarado, \& Charo-Alonso, 2009; Yilmaz \& Ogutcu, 2014), monoglycerides (Chen \& Terentjev, 2009; Co \& Marangoni, 2012; Lopez-Martinez, Charo-Alonso, Marangoni, \& Toro-Vazquez, 2015; Lopez-Martinez et al., 2014; Valoppi et al., 2017) and sorbitans (Murdan, Gregoriadis, \& Florence, 1999; Pernetti, van Malssen, Kalnin, \& Floter, 2007; Sanchez, Franco, Delgado, Valencia, \& Gallegos, 2011) are some of the materials that have been used to gel edible oils.

Sorbitan esters, also called spans, are non-ionic surfactants, which are hydrophobic in nature and that can act as emulsifiers and structuring agents in organic solvents. In particular, Span 60 and Span 65 are esters of sorbitan and stearic acid, differing by the number of alkyl chains (esterification degree) and presenting one and three tails, respectively. In the last decade some studies reported their ability to produce organogels with edible oils, their application as fat replacer and mainly as delivery systems (Barbut, Wood, \& Marangoni, 2016; Kamble, Udapurkar, Nakhat, Yeole, \& Biyani, 2011; Singh, Pramanik, Ray, \& Pal, 2015). Glyceryl monostearate (GM) and tristearate (GT) are esterification products of glycerin and stearic acid that also differ by the presence of one and three alkyl chains, respectively. A number of studies report the use of GM to produce organogels. On the other hand, GT is usually combined with other organogelator since it is not classified as an amphiphilic molecule due the complete esterification of its hydrophilic head. Despite of this GT could be used as a potential gelator of organic solvents due its hardening properties (Sahri \& Idris, 2010). Therefore, the main contribution of the present work is the improvement in the knowledge about the mechanism of organogel structure formation with food-grade ingredients, their structural and mechanical properties, since there are few works trying to unravel, explain and compare the formation mechanism of organogels with these organogelators. Our work evaluates the effect of gelators with different hydrophilic heads (glyceryl and sorbitan group) and hydrophobic tails (mono- or tri-stearic chains) and the size of oils' carbon chain (i.e. C8-C10 or C16-18) on organogel properties. The developed organogels were evaluated by oscillatory rheology in order to understand how the molecular organization influences their mechanical behavior. These properties were correlated with their crystalline structures, which were studied through small-angle X-ray scattering (SAXS) and polarized microscopy.

\section{Materials and methods}

\subsection{Raw materials}

Glyceryl tristearate (GT) (>98.0\%), sorbitan tristearate (ST) and sorbitan monostearate (SM) of technical grade were purchased from Sigma Aldrich Chemical Co. Ltd. (USA) and glyceryl monostearate (GM) (>95.0\%) was obtained from Alfa Aesar (USA). Medium-chain triglycerides (MCT, Neobee 1053; composed by $55 \%$ of C 8 and $44 \%$ of C10 fatty acids) was kindly supplied by Stepan Lipid Nutrition (USA) and high-oleic sunflower oil (LCT, $0.1 \%$ of $\mathrm{C} 14: 0,3.8 \%$ of $\mathrm{C} 16: 0,3.3 \%$ of $\mathrm{C} 18: 0,80.1 \%$ of $\mathrm{C} 18: 1,10.7$ of $\mathrm{C} 18: 2,0.3 \%$ of $\mathrm{C} 18: 3,0.4 \%$ of $\mathrm{C} 20: 0$ and $0.1 \%$ of $\mathrm{C} 20: 1$ ) was donated by Cargill (Brazil). Table 1 presents the structure, hydrophilic-lipophilic balance (HLB), melting point and molecular weight of the gelators used.

\subsection{Organogels production}

Organogels were prepared in $8 \mathrm{~cm}$ height $\times 2 \mathrm{~cm}$ diameter tubes with screw caps by heating the mixture of oil phase (MCT or LCT) and the gelator $(5,10,15,20$ and $25 \% \mathrm{w} / \mathrm{w})$ at $80^{\circ} \mathrm{C}$ during 30 min under magnetic stirring. Afterwards samples were allowed to cool down at $25{ }^{\circ} \mathrm{C}$ under quiescent conditions. The concentrations were chosen based on the critical gelation concentration for the gelators and the type of oil phases used (Table 1S). Gelator concentration above 15\% (w/w) (upper limit to be considered as an organogel) was evaluated to help understand the interactions between components and network formation. The samples were stored at $25 \pm 2{ }^{\circ} \mathrm{C}$ at least during $24 \mathrm{~h}$ before being analyzed. Organogels produced with MCT and LCT will be called OMCT and OLCT, respectively.

Table 1

Hydrophilic-lipophilic balance (HLB), melting point, molecular weight values and chemical structure of the gelators used for organogels production.

\begin{tabular}{|c|c|c|c|c|}
\hline Organogelator & HLB & Melting point ${ }^{\mathrm{a}}\left({ }^{\circ} \mathrm{C}\right)$ & Molecular weight $^{\mathrm{a}}(\mathrm{Da})$ & Chemical structure \\
\hline Glyceryl tristearate (GT) & 2.0 & 55 & 891.5 & \\
\hline Glyceryl monostearate (GM) & 3.8 & $58-59$ & 358.57 & \\
\hline Sorbitan tristearate (ST) & 2.1 & 53 & 963.54 & \\
\hline Sorbitan monostearate (SM) & 4.7 & $54-57$ & 430.62 & \\
\hline
\end{tabular}

${ }^{a}$ Values obtained from suppliers and Hepworth (2006). 


\subsection{Polarized microscopy}

Organogels samples at $80{ }^{\circ} \mathrm{C}$ were placed on a glass slide and stored at $25^{\circ} \mathrm{C}$ during $24 \mathrm{~h}$ before analyses. Images were obtained under a polarized light microscope (Olympus System Microscope model BX 50, Olympus America Inc., Center Valley, PA, USA) equipped with a digital camera (Nikon DS-Ri1, Melville, NY, USA) and using lens Nikon AZ Plan Apo4x (Melville, NY, USA). Pictures were taken at a magnification of $320 \times$.The software used was NIS-Elements microscope imaging software (Nikon, USA). The samples after preparation at $80^{\circ} \mathrm{C}$ were poured directly in the support and conditioned at $25^{\circ} \mathrm{C}$ during $24 \mathrm{~h}$ before being analyzed at room temperature.

\subsection{Rheological analyses}

Rheological measurements were performed at $25^{\circ} \mathrm{C}$ using a Physica MCR301 (Anton Paar, Graz, Austria) rheometer equipped with a stainless steel cone-plate geometry ( $50 \mathrm{~mm}, 2^{\circ}$ angle, truncation $208 \mu \mathrm{m}$ ). The samples were handled gently to avoid structural damage. Flow curves were obtained by a three-shear rate sweeps (up-down-up) program using a continuous ramp and shear rate range between 0 and $300 \mathrm{~s}^{-1}$. The three steps program was carried out in order to eliminate the time-dependence, allowing the system to reach the steady state. The apparent viscosity at low shear rate $\left(3 s^{-1}-\eta_{3}\right)$ was evaluated from curve 1 (unsteady-state) since the structure was less disturbed at this condition. Qualitative thixotropy was evaluated from hysteresis area between curves 1 (unsteady-state) and 3 (steady-state). The viscoelastic properties were evaluated by oscillatory measurements, using a frequency sweep between 0.1 and $10 \mathrm{~Hz}$ within the linear viscoelasticity domain (1\% deformation). Storage $\left(G^{\prime}\right)$, loss $\left(G^{\prime \prime}\right)$ moduli and tan $\delta$ were evaluated.

\subsection{Small angle X-ray scattering}

Small angle X-ray scattering (SAXS) measurements were performed at room temperature using the beamline of the National Synchrotron Light Laboratory (LNLS, Campinas, Brazil). The beamline is equipped with an asymmetrically cut and bent silicon (111) monochromator that yields a monochromatic $(\lambda=1.54 \AA$ ) and horizontally focused beam. A position-sensitive X-ray detector and a multichannel analyzer were used to record the SAXS intensity, $\mathrm{I}(\mathrm{q})$, as a function of modulus of scattering vector $q=(4 \pi / \lambda) \sin (\theta / 2), \theta$ being the scattering angle. Each SAXS pattern corresponds to a data collection time of $100 \mathrm{~s}$. Bragg law was used for the determination of the structure factor that represents the crystal organization through the relative position of the diffraction peaks. For a lamellar structure the distance between peaks has the relation 1:2:3:4. The lattice parameter $d$ (distance between the structures' periodicity) of the lamellar structures was obtained from the position $(\mathrm{q})$ of the first (and more intense) diffraction peak using the relation $d=2 \pi / \mathrm{q}$. The form factor $(-1,-2$, or -4 for rod, disk, or a sphere, respectively) was determined, evaluating the slope of the log-log plot at small angles (Roe, 2000). ATSAS software was used for analysis of SAXS data (Petoukhov et al., 2012).

\section{Results and discussion}

\subsection{Visual observations and polarized microscopy}

Preliminary visual observation was used to identify self-supported organogels by simple tube flipping (Patel, Babaahmadi, Lesaffer, \& Dewettinck, 2015). The systems that did not flow under the influence of gravity were named as organogels. For MCT-based (OMCT) systems, organogels were obtained with at least 3\% (w/w) GM, 8\% (w/w) ST and $12 \%(\mathrm{w} / \mathrm{w})$ for GT and SM. For organogels produced with LCT (OLCT) as oil phase, organogels were obtained at the same concentrations for GM and ST while in the cases of GT and SM those concentrations were reduced to $10 \%(\mathrm{w} / \mathrm{w})$ of organogelators (Table 1S). However, in order to guarantee the stability of organogels the range of concentrations was increased to between 5 and $25 \%$ of organogelator. This wide range was used because there is not a concern about the maximal concentration of organogelator to classify the system as organogel. However, it is generally accepted that a gel is a semi-solid material composed of low concentrations ( $<15 \%)$ of organogelator (Vintiloiu \& Leroux, 2008), although a high number of works had used higher organogelator concentrations (Lupi et al., 2016; Penzes, Csoka, \& Eros, 2004; Singh et al., 2015). In addition, the use of a wider gelator concentration will allow to understand the role of molecular characteristics of gelator on the organogels properties.

Fig. 1 shows the optical micrographs of OMCT and OLCT at the lowest $(5 \% \mathrm{w} / \mathrm{w})$ and highest $(25 \% \mathrm{w} / \mathrm{w})$ concentration of gelator. It is clear for all samples the occurrence of birefringence of crystalline structures. The increase of gelator concentration promotes the reorganization of the crystals leading to different microscopy images according to the type and concentration of gelator used. SAXS spectra (section 3.3) shows the presence of a lamellar structure for all the structures; also for the ones at concentrations lower that the critical gelation concentrations. When the mixture was cooled the crystal phase is formed leading to the formation of a polydomain crystal network. Organogel formation occurs into three steps: initial crystal nucleation, the repeating crystalline branching and the final crystal growth (Yu, Lin, Yub, \& Liu, 2015). If crystal growth is restricted to two dimensions, then platelet-like structures are obtained. Structures formed at low gelator concentrations can be related with this two-dimensional mechanism that gives rise to nucleation and growth of the crystal surface before gel formation. Gelation requires the creation of a continuous network, often achieved by the crystal cross-linking or entanglement, trapping the mobile phase. With the increase of the gelator concentration this process continues until the formation of a three-dimensional structure immobilizing all the oil phase (Huang, Terech, Raghavan, \& Weiss, 2005; Rogers \& Kim, 2011; Rogers, Pedersen, \& Quaroni, 2009).

In general, sorbitan hydrophilic head produced more compact structures when compared with the glycerol-based ones. In addition, the presence of one hydrophobic tail (monostearate) leds to the formation of a more dense crystalline texture (more pronounced birefringence) when compared with the ones with tristearate (e.g. Fig. 1a and i). Furthermore, for GT it is clear that the increase of gelator concentrations favors the crystallization process leading to formation of crystals in a greater number (confirmed by the increase of the birefringence). On the other hand, organogels produced using GM (Fig. 1e, f, g and h) presented a crystalline microstructure, similar to the one presented by other authors (Batte, Wright, Rush, Idziak, \& Marangoni, 2007). However, the micrographs also showed that the use of different oils affect the crystal formation as can be seen in Fig. 1. The oil polarity and unsaturation degree exert effect on its ability to form H-bonds with organogelator molecules (Gravelle et al., 2016). For OLCT the polarized micrographs showed that at $5 \%(\mathrm{w} / \mathrm{w})$ of gelator the crystal structure network is formed in all cases. However, for both oil types the increase of concentration leads to a morphological transition where the organogels' structure becomes more compact, with their matrix being more homogeneous explained by the presence of a dense and self-supported crystalline structure. Furthermore, OLCT micrographs seem more dense and birefringent than OMCT.

\subsection{Rheological measurements}

Elastic $\left(G^{\prime}\right)$ and viscous $\left(G^{\prime \prime}\right)$ moduli profiles of OMCT and OLCT are shown in Figs. 2 and 3, respectively, and show dependence on the type and concentration of gelator used. Rheological measurements showed similar behavior for organogels produced with both MCT and LCT. The $G^{\prime}$ and $G^{\prime \prime}$ increased from 5 to $25 \%(\mathrm{w} / \mathrm{w})$ of gelator, since the network was reinforced by the gelator structure and the oil phase. 
$5 \%$

ОМСT
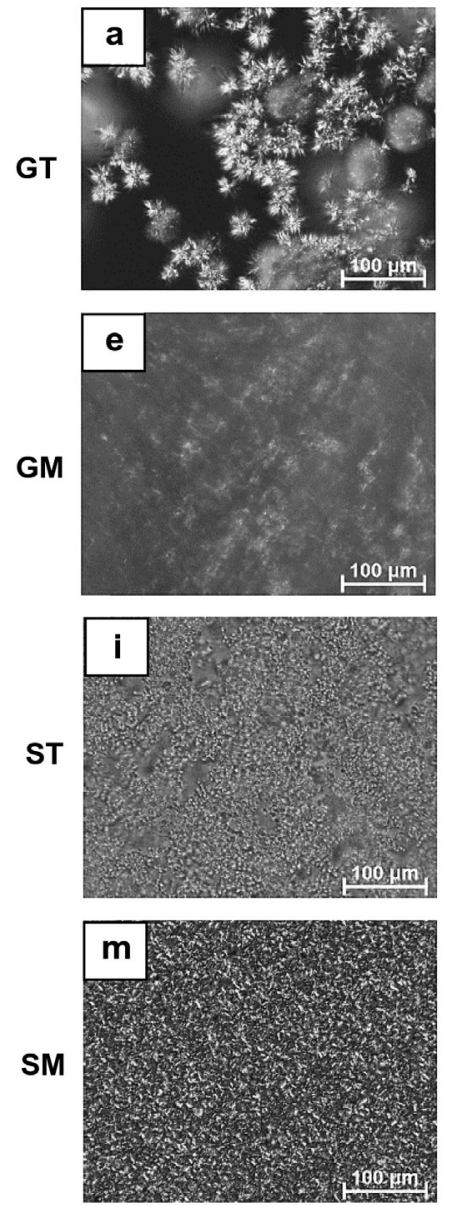

$25 \%$

OMCT
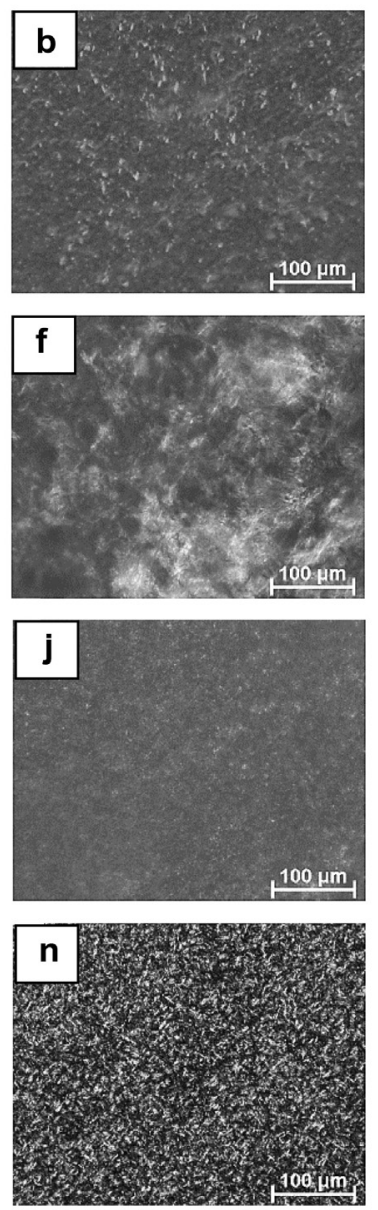

$5 \%$

OLCT
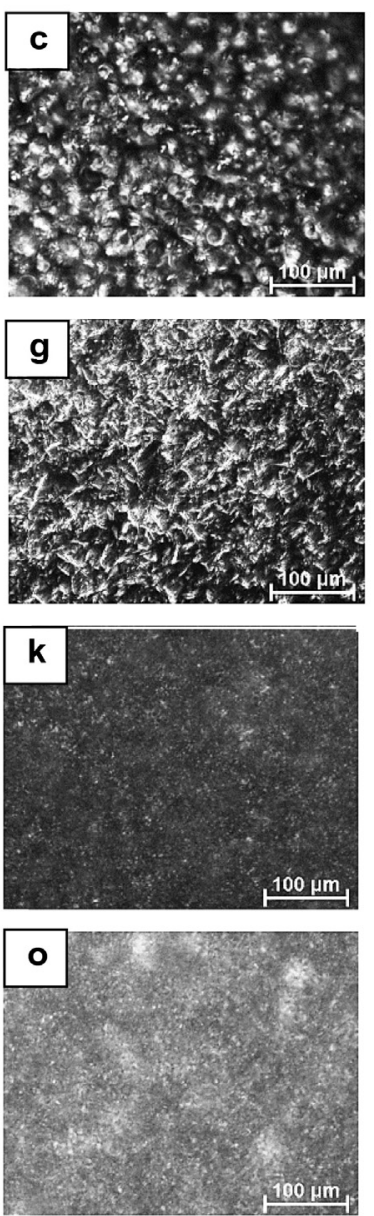

$25 \%$

OLCT
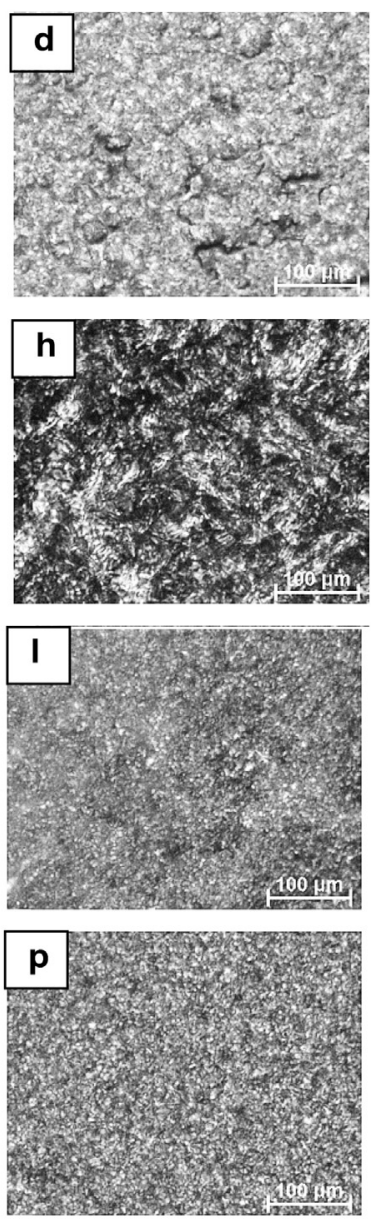

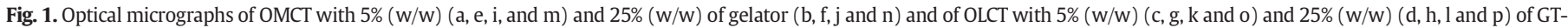
glyceryl tristearate (a, b, c and d), GM-glyceryl monostearate (e, f, g and h), ST-sorbitol tristearate (i, j, k and l) and SM-sorbitol monostearate (m, n, o and p).

However, at the lowest gelator concentration ( $5 \% \mathrm{w} / \mathrm{w})$ only GM stood out from the other organogelators moduli for more than a decade. This is in agreement with visual observations, where GM was the only one forming self-supported gels at this concentration, while for the systems at 5\% (w/w) using GT, ST and SM a structured liquid behavior was observed (flowed slowly) (Rocha et al., 2013).

The addition of 10 and $15 \%(\mathrm{w} / \mathrm{w})$ of gelator to OLCT and OMCT respectively, lead to self-supporting gels and a common behavior was observed for elastic and viscous moduli that was that glycerol-based gelators showed higher elastic moduli $\left(G^{\prime}\right)$ than sorbitan-based gels ( GM $>$ GT $>$ SM $>$ ST). Glycerol hydrophilic head is smaller and less bulky in comparison to sorbitan's, since glyceryl head is a linear structure and sorbitans present a carbon ring that confers to them a large and rigid conformation (Davidovich-Pinhas, Barbut, \& Marangoni, 2015b; Gravelle, Barbut, Quinton, \& Marangoni, 2014). The less bulky conformation allows the approximation of the molecules that can interact more closely with its neighbors. These interactions are short-range weak forces (hydrophobic interactions, dipole moment and H-bonding) that led to formation of stronger elastic structures and resistance to deformation (Toro-Vazquez et al., 2013). In fact, at the molecular level, the size and chemical nature of the head group will influence the arrangement of lamellar structures (as found in SAXS analyses - see section 3.4). Regarding the hydrophobic tail of the gelators, gels produced with monostearate tended to be stronger than those produced with tristearate at low gelator concentrations, since the arrangement of the tristearate molecule is more rigid and presumably prevents sterically the entanglement of tails (Abraham et al., 2012; Sato, Yoshimoto, Suzuki, Kobayashi, \& Kaneko, 1990; Toro-Vazquez et al., 2013). However, this tendency is inverted at higher gelator concentrations and the gel strength presents a new order of magnitude: $\mathrm{GT}>\mathrm{GM}>\mathrm{SM}>\mathrm{ST}$. The presence of more hydroxyl groups in the GM molecule than in the GT molecule renders the former more hydrophilic behavior, eventually leading to the establishment of a higher number of e.g. hydrogen bonds and consequently to the formation of a stronger gel network, as occurred at lower concentrations. On the other hand, a sterical effect of the higher molecular weight of hydrophobic portion (three tails) of tristearate with the increase of gelator concentration was also observed. Moreover, the van der Waals forces between the alkyl chains became relevant and in association with the glycerol hydrophilic head aggregation produced stronger gels (Toro-Vazquez et al., 2013). In addition, this inversion occurred at lower concentrations of gelator for gels prepared with LCT than for MCT. Thus, the formation of harder or softer structures will depend on the combination of physical interactions and sterical effects between gelator and oil.

The effect of oil composition can be related to its TAG composition, including chain length and unsaturation degree (Laredo, Barbut, \& Marangoni, 2011; Valoppi et al., 2017). Oils with long chain, higher viscosity and lower dielectric constant leads to organogels with increased firmness and rheological properties. Moreover, the ability of MCT's to make dipole-dipole rotation affect the crystallization 


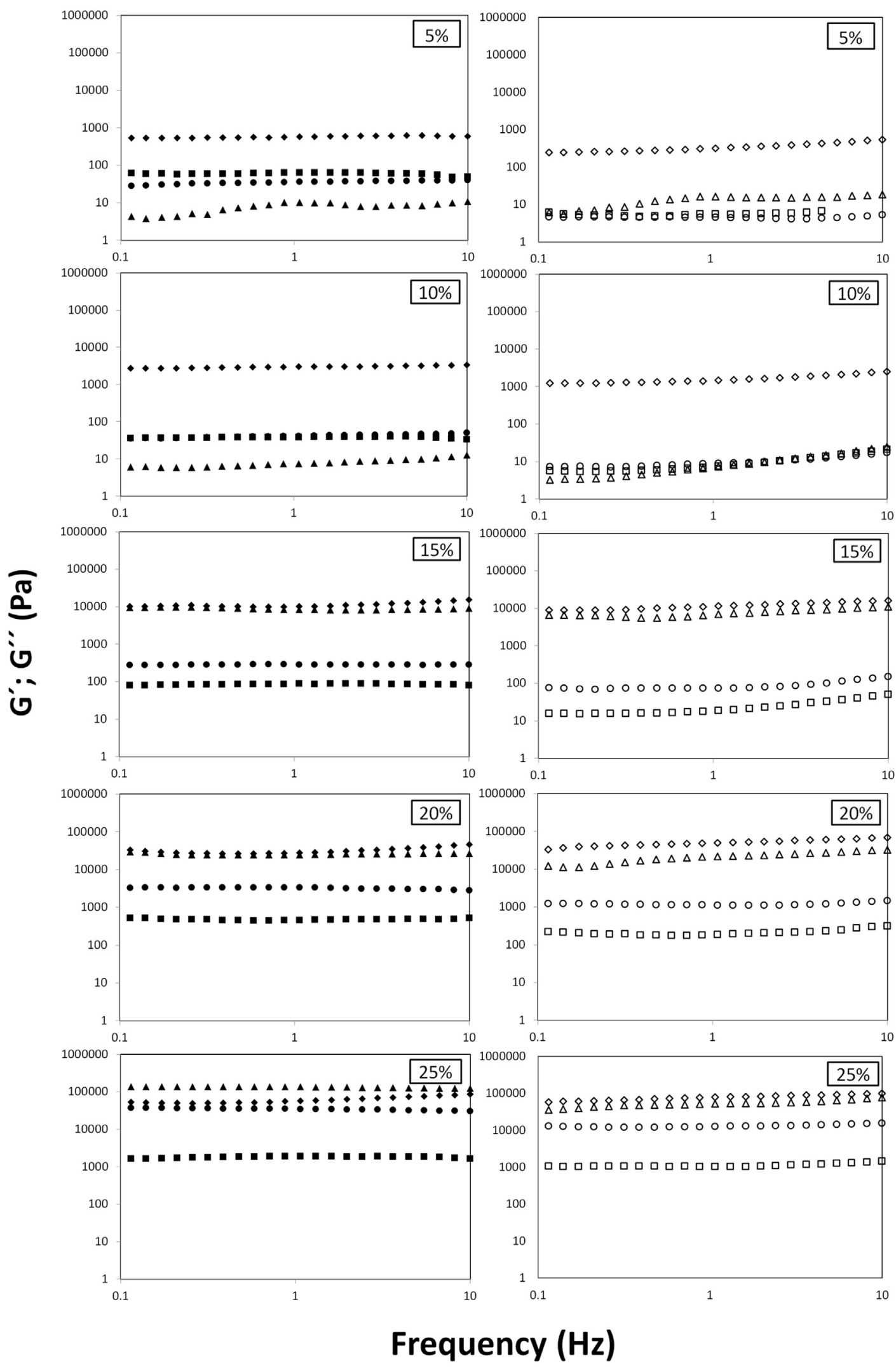

Fig. 2. Storage ( $G^{\prime}$ - full symbols) and loss ( $G^{\prime \prime}$ - empty symbols) moduli as function of frequency for OMCT produced with GT $(\bullet)$, GM $(\bullet)$, ST (®) and SM $(\bullet)$ as gelator and for increasing concentrations of gelator $(5 \%, 10 \%, 15 \%, 20 \%$ and $25 \%, w / w)$.

behavior (Valoppi et al., 2017). The prevailing fatty acid composition of MCT is C8:0 (55\%) and C10:0 (44\%) while for LCT is C18:1 (80\%), which is in agreement with this assertion, since higher moduli $\left(\mathrm{G}^{\prime}\right.$ and $G^{\prime \prime}$ ), more structured and birefringent systems were observed for OLCT. Moreover, the LCT used also present prevailing fatty acid with one unsaturation. The greater unsaturation degree led to higher 


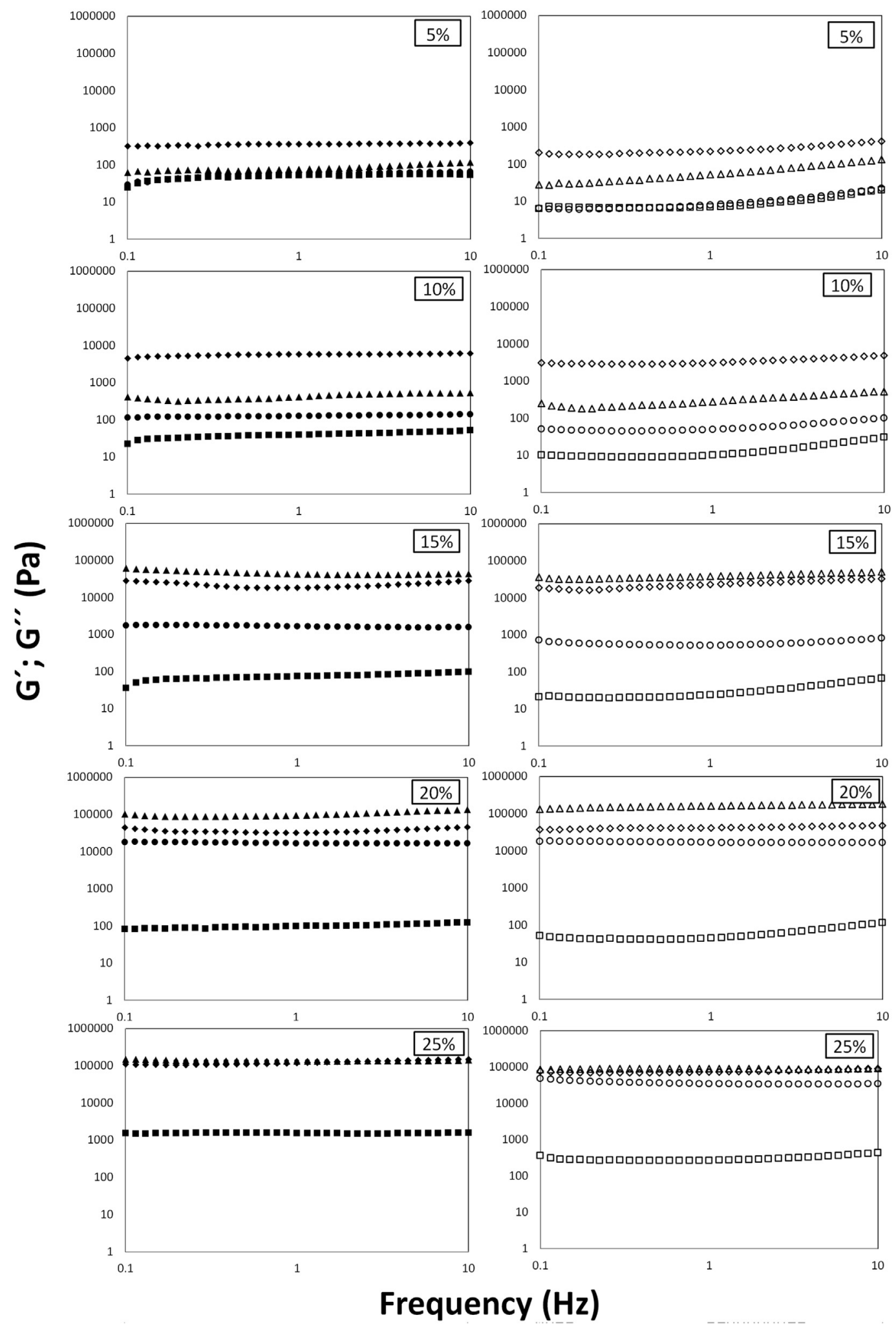

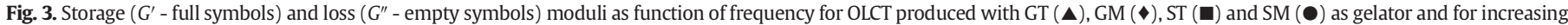
concentrations of gelator $(5 \%, 10 \%, 15 \%, 20 \%$ and $25 \%, \mathrm{w} / \mathrm{w})$.

conformational freedom and a higher molar volume of the solvent. Moreover, the higher unsaturation degree led to a more crooked spatial arrangement, decreasing the interaction energy and the oil behave as more hydrophobic (Phan, Harwell, \& Sabatini, 2010). This higher hydrophobicity could be more efficient to solubilize the organogelator tails and to force the aggregation of the hydrophilic head. All these characteristics would facilitate the formation of a greater number of junction zones, producing stronger gels (Laredo et al., 2011).

Organogels produced using ST or SM with MCT or LCT showed a gellike behavior with the predominance of $\mathrm{G}^{\prime}$ over $\mathrm{G}^{\prime \prime}$ and the independence of frequency over a very broad frequency range. On the other 
hand, $G^{\prime}$ and $G^{\prime \prime}$ values of organogels produced with glycerol-based gelators were very similar to each other, showing $\tan \delta$ close to the unity (Fig. 4), which cannot be considered a true gel. Despite of this, systems formed by GM and GT showed higher moduli values and a visual appearance of more structured gels in comparison to sorbitan-based organogels. It is also possible to observe that in some cases $G^{\prime}$ and $G^{\prime \prime}$ present a different behavior as the frequency increases, being the $G^{\prime}>$ $G^{\prime \prime}$ until a given frequency, after which $G^{\prime \prime}$ overtakes $G^{\prime}$ (cross-over observed by $\tan \delta$ at the unit). The frequency at which this crossover happens can be called by longest relaxation time (Mezzenga et al., 2005 ) and is observed for lower concentrations (5 and 10\%) of GT in OMCT and OLCT.

Fig. 1S shows the flow curves for OMCT and OLCT using $5 \%(w / w)$ of gelator. Apparent viscosity at low shear rate $\left(3 \mathrm{~s}^{-1}\right)$ was evaluated from unsteady-state curves or minimal disturbance of the structure. Organogels showed the same behavior observed for frequency sweep
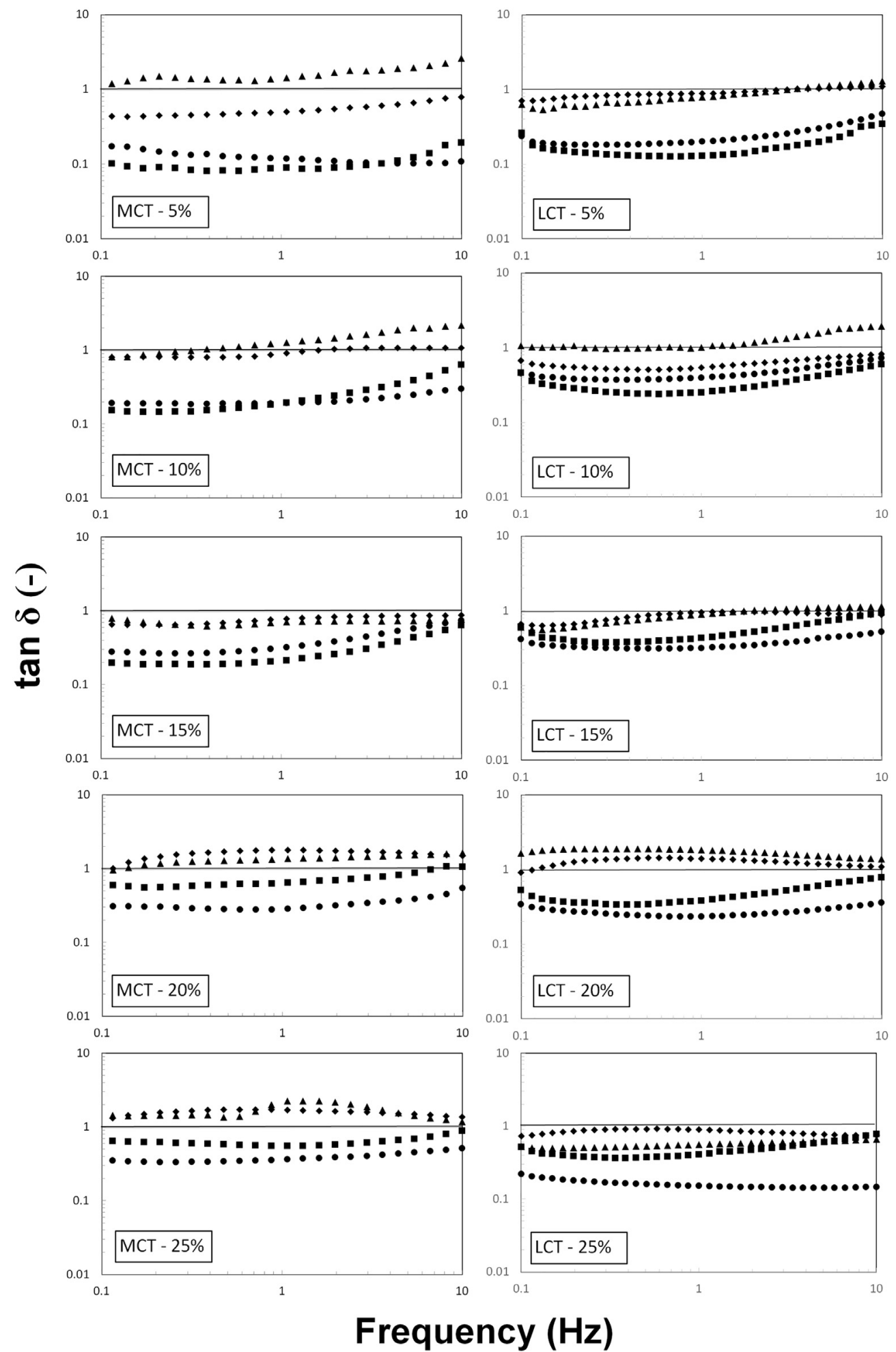

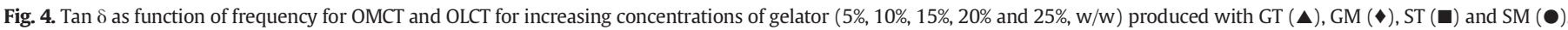


and those produced with glycerol-based molecules presented higher viscosity values ( $\mathrm{GM}>\mathrm{GT}>\mathrm{SM}>\mathrm{ST})$. Moreover, it was clear the influence of the oil type on this parameter, having OLCT almost twice the viscosity of OMCT. At lower gelator concentrations (5 and 10\% $\mathrm{w} / \mathrm{w}$ ) and using MCT as oil phase only the samples produced using GM as gelator showed a stress overshoot at low shear rates while for the organogel produced using ST as gelator and LCT as oil phase this behavior was observed only for concentrations of 25\% (Table $2 \mathrm{~S}$ ).This phenomenon (a peak of shear stress indicated by arrows in Fig. 1S) can be explained by the force needed to start to break the elastic network structure subjected to flow (Rocha et al., 2013). High values of this parameter can be related to stronger network and thixotropic behavior. All the organogels presented thixotropy, which was verified by the hysteresis between unsteady and steadystate curves (curves 1 and 3, respectively). This evaluation can be qualitatively related to the shear time-dependence (thixotropy) of the material, where it is zero for a time-independent system (Steffe, 1996). Results showed that glycerol-based organogels presented higher hysteresis values (qualitative thixotropy) than sorbitan-based organogels in the following order for both oil: GM $>$ GT $>$ SM $>$ ST (Fig. 5). It is important to note that this behavior was the same observed for frequency sweeps and viscosity values that are also an indicative of the gel strength. Higher hysteresis or thixotropy can be related to more structured gels with stronger network. The inversion between GM and GT at higher gelator concentration did not appear in the flow curves, when comparing rheological properties obtained under small or large deformation (oscillatory and flow curves, respectively). This result reinforce that the interaction between the hydrophilic head is the main responsible for the structure formation and strengthening of organogels. Moreover, thixotropic values of OLCT are higher when compared with OMCT at the same concentrations, confirming that the higher molecular weight and unsaturation degree play an important role on the network structure.
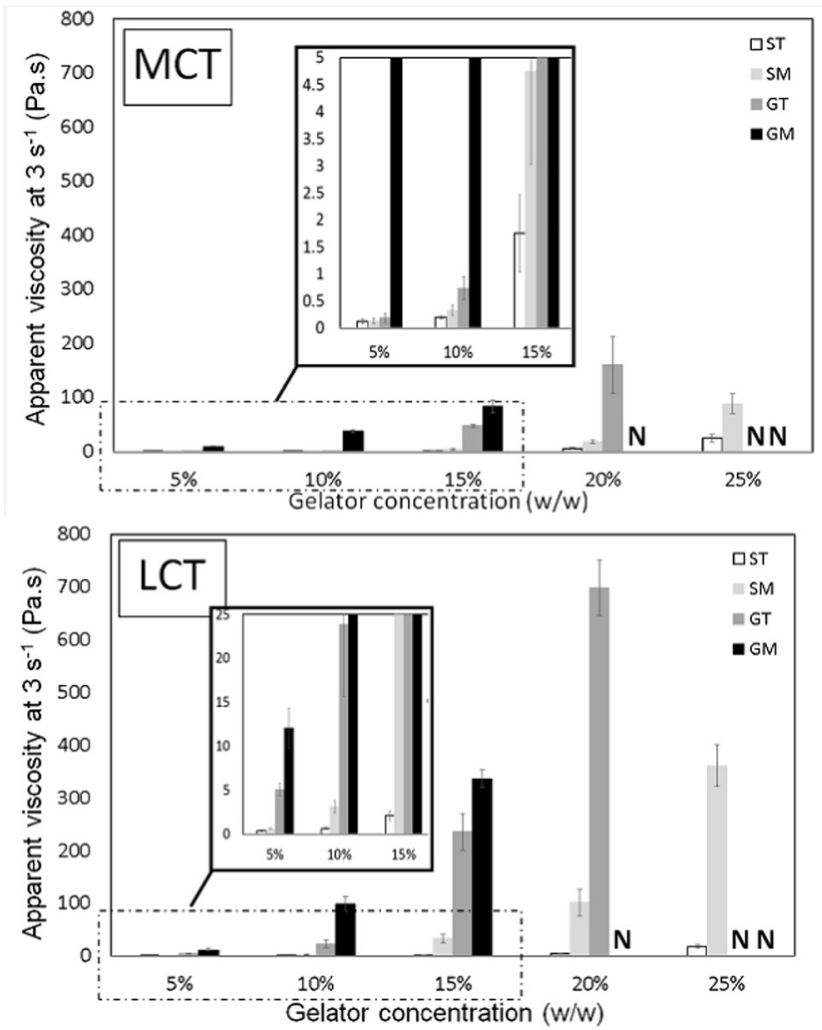

\subsection{Small-angle $X$-ray scattering}

Small-angle X-ray scattering (SAXS) spectra are characterized by sharp peaks followed by weaker reflections giving information on crystal structure. SAXS profiles when using GT as gelator presents two peaks in the relation 1:2 that is typical of lamellar structures. Moreover, the differences between the SAXS parameters ( $d$-spacing - distance between the lamellas) of the organogels were related to properties and concentrations of gelator and oil. At concentrations below $15 \%(w / w)$ the second peak presents a very low intensity, increasing for higher concentrations, due to the presence of a high number of crystal structures. Fig. $2 \mathrm{~S}$ shows the log-log plots of SAXS curves of the organogels (for a concentration of 15\%) that provide an indication of the type of structure developed (slope for low angles $q<0.08 \AA$ ). All organogels presented a slope ranging between $d^{-2}$ and $d^{-3}$ indicating the presence of a flat-type structure (Roe, 2000). The same behavior was observed for the organogels produced with both MCT and LCT oils. Polarized microscopy results (Fig. 1) showed that the formation of a three-dimensional crystal structure for high gelator concentrations is clear, but it is still possible to see the presence of crystals at low concentrations. The intensities of the peaks increased for higher concentrations of gelators, being this difference clear when the concentrations lead to the formation of a gel: e.g. for organogels using GT as gelator the second peak was more intense for concentrations of $15 \%(w / w)$ or higher, which are the concentrations where the organogels behave as gel-like system $\left(G^{\prime}>G^{\prime \prime}\right)$. This result indicates that a three-dimensional crystal network is effectively formed.

OMCT using GM as gelator showed lamellar structures with $d=$ 48.06 Å for the lowest concentration of gelator (5\%) (Table 2). For increasing concentrations of gelator $(10,15$ and $20 \% \mathrm{w} / \mathrm{w})$ distances around 66 Å were observed, also representing a lamellar structure. The change of $d$-spacing could be explained by the modification of the
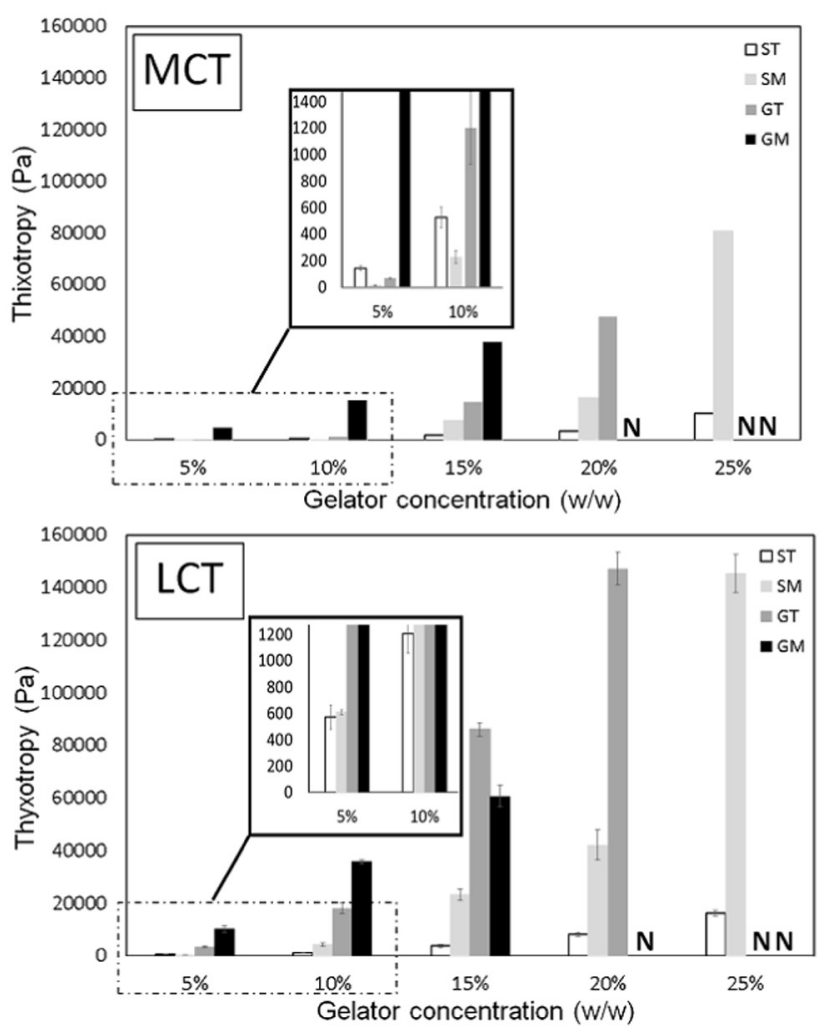

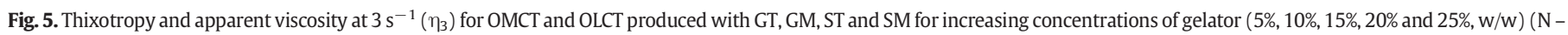
not performed). 
Table 2

Structural parameters of organogels from MCT and LCT evaluated by small-angle X-ray scattering.

\begin{tabular}{|c|c|c|c|c|c|c|}
\hline \multirow[b]{2}{*}{ Gelator } & & \multicolumn{5}{|c|}{ Medium-chain triglycerides (MCT) } \\
\hline & & $5 \%$ & $10 \%$ & $15 \%$ & $20 \%$ & $25 \%$ \\
\hline \multicolumn{7}{|c|}{ Small angle diffractions $(\AA)$} \\
\hline \multirow[t]{2}{*}{ GT } & 001 & 44.17 & 44.58 & 44.73 & 44.72 & 44.82 \\
\hline & 002 & 21.29 & 21.63 & 21.49 & 21.70 & 22.50 \\
\hline \multirow[t]{2}{*}{ GM } & 001 & 48.06 & 65.19 & 66.70 & 67.80 & 48.19 \\
\hline & 002 & 23.35 & 31.23 & 31.38 & 32.56 & 24.14 \\
\hline \multirow[t]{2}{*}{ ST } & 001 & 51.51 & 51.55 & 51.52 & 51.86 & 51.76 \\
\hline & 002 & 23.67 & 25.78 & 25.73 & 25.83 & 25.82 \\
\hline \multirow[t]{2}{*}{ SM } & 001 & 59.06 & 61.88 & 48.17 & 48.13 & 48.10 \\
\hline & 002 & 28.55 & 30.23 & 22.41 & 22.46 & 24.40 \\
\hline \multicolumn{7}{|c|}{ Long-chain triglycerides (LCT) } \\
\hline \multirow[t]{2}{*}{ GT } & 001 & 44.31 & 44.69 & 44.90 & 44.46 & 44.62 \\
\hline & 002 & 22.77 & 22.33 & 23.07 & 22.89 & 22.36 \\
\hline \multirow[t]{2}{*}{ GM } & 001 & 47.75 & 47.80 & 51.54 & 52.13 & 47.72 \\
\hline & 002 & 23.89 & 23.82 & 25.76 & 26.00 & 23.81 \\
\hline \multirow[t]{2}{*}{ ST } & 001 & 52.08 & 52.10 & 52.57 & 52.40 & 51.31 \\
\hline & 002 & 25.62 & 25.57 & 24.86 & 25.79 & 25.72 \\
\hline \multirow[t]{2}{*}{ SM } & 001 & 56.76 & 64.82 & 62.61 & 63.96 & 63.96 \\
\hline & 002 & 28.95 & 21.44 & 31.56 & 29.62 & 29.62 \\
\hline
\end{tabular}

structure due to the increase of gelator concentration (Zetzl, Ollivon, \& Marangoni, 2009). It is interesting to note that for the highest concentration of gelator $(25 \% \mathrm{w} / \mathrm{w})$ the structure changes again, since the formation of a lamellar structure with a $d$-spacing similar to the one obtained for $5 \%(w / w)$ of gelator (Table 2 ) was observed. For OLCT the increase of concentration ( 15 and $20 \% \mathrm{w} / \mathrm{w}$ ) also leads to a modification of the $d$-spacing with values around 52 , indicating also a change in the organogel structure in this concentration range $(15-20 \% \mathrm{w} / \mathrm{w})$. Organogels produced with ST and SM as gelators presented similar lamellar structures for both types of oil (Table 2). For organogels of ST both OMCT and OLCT showed similar $d$-spacing values; in the case of SM the $d$-spacing decreased for concentrations of 15,20 and $25 \%$ (w/w) of organogels using MCT as oil. Overall, the mean point when comparing the $d$-spacing between organogels with different types of gelators and oils are that: a) it is clear the presence of lamellar structures in all cases; b) sorbitol-based gelators led to higher $d$-spacing values, which could be related with the size of the hydrophilic head, as explained before (see section 3.1).

\section{Conclusions}

The use of different gelators with different hydrophilic heads (glyceryl and sorbitan group) and hydrophobic tails (mono- or tristearic chains) in the production of organogels influenced their structure and properties. In general, rheological properties increased for higher concentrations of gelator justified by the effect of a more structured network formation. Nevertheless, this increase is not linear for all the gelators. Both glyceryl-based gelators (i.e. GM and GT) produced the most structured organogels with higher values of rheological parameters attributed to the presence of the glyceryl head group. Regarding the influence of the hydrophobic tail, it is clear that the use of monostearate led to the formation of stronger organogels with higher spacing between lamellae. However, the evaluation of the organogelator concentration effect showed that there is a threshold where the gelation mechanism changes. At concentration above $15 \%(\mathrm{w} / \mathrm{w})$ the sterical effects became relevant, increasing the gel strength. At last, the type of oil used (LCT or MCT) also exerted influence on these properties. Oleogels produced with LCT were stronger and gelled at lower concentration of organogelator than MCT. Based on the results presented here it can be stated that it is possible to tailor the properties and functionality of organogels through combining different gelators and oils in order to obtain a product with desirable characteristics. The use of organogels with tunable properties can be very interesting in order to replace trans and saturated fats in food products, with a low impact on food structure and consumer perception.

\section{Acknowledgments}

The authors thank the FCT Strategic Project of UID/BIO/04469/2013 unit, the project RECI/BBB-EBI/0179/2012 (FCOMP-01-0124-FEDER027462) and the project "BioInd - Biotechnology and Bioengineering for improved Industrial and Agro-Food processes", REF. NORTE-070124-FEDER-000028 co-funded by the Programa Operacional Regional do Norte (ON.2 - O Novo Norte), QREN, FEDER. The financial support of CAPES/FCT Project "Nanotechnological systems based in biocompatible ingredients: characterization, controlled release and in vitro digestion" (CAPES/FCT n ${ }^{\circ} 348 / 13$ ) and CNPq (Universal 479459/2012-6) are gratefully acknowledged. The authors also thank the Brazilian Synchrotron Light Laboratory (LNLS) for the opportunity to carry out SAXS measurements.

\section{Appendix A. Supplementary data}

Supplementary data to this article can be found online at http://dx. doi.org/10.1016/j.foodres.2017.03.021.

\section{References}

Abdallah, D. J., \& Weiss, R. G. (2000). Organogels and low molecular mass organic gelators. Advanced Materials, 12(17), 1237.

Abraham, S., Lan, Y., Lam, R. S., Grahame, D. A., Kim, J. J., Weiss, R. G., \& Rogers, M. A. (2012). Influence of positional isomers on the macroscale and nanoscale architectures of aggregates of racemic hydroxyoctadecanoic acids in their molecular gel, dispersion, and solid states. Langmuir, 28(11), 4955-4964. http://dx.doi.org/10.1021/ la204412t.

Barbut, S., Wood, J., \& Marangoni, A. (2016). Potential use of organogels to replace animal fat in comminuted meat products. Meat Science, 122, 115-162.

Batte, H. D., Wright, A. J., Rush, J. W., Idziak, S. H. J., \& Marangoni, A. G. (2007). Phase behavior, stability, and mesomorphism of monostearin-oil-water gels. Food Biophysics, 2(1), 29-37. http://dx.doi.org/10.1007/s11483-007-9026-7.

Chen, C. H., \& Terentjev, E. M. (2009). Aging and metastability of monoglycerides in hydrophobic solutions. Langmuir, 25(12), 6717-6724. http://dx.doi.org/10.1021/ la9002065.

Co, E. D., \& Marangoni, A. G. (2012). Organogels: An alternative edible oil-structuring method. Journal of the American Oil Chemists' Society, 89, 749-780.

Davidovich-Pinhas, M., Barbut, S., \& Marangoni, A. G. (2015a). The gelation of oil using ethyl cellulose. Carbohydrate Polymers, 117, 869-878. http://dx.doi.org/10.1016/j. carbpol.2014.10.035.

Davidovich-Pinhas, M., Barbut, S., \& Marangoni, A. G. (2015b). The role of surfactants on ethylcellulose oleogel structure and mechanical properties. Carbohydrate Polymers, 127, 355-362. http://dx.doi.org/10.1016/j.carbpol.2015.03.085.

Davidovich-Pinhas, M., Barbut, S., \& Marangoni, A. G. (2016). Development, characterization, and utilization of food-grade polymer oleogels. Annual Review of Food Science and Technology, 7, 65-91. http://dx.doi.org/10.1146/annurev-food041715-033225.

Gravelle, A. J., Barbut, S., Quinton, M., \& Marangoni, A. G. (2014). Towards the development of a predictive model of the formulation-dependent mechanical behaviour of edible oil-based ethylcellulose oleogels. Journal of Food Engineering, 143, 114-122. http://dx.doi.org/10.1016/j.jfoodeng.2014.06.036.

Gravelle, A. J., Davidovich-Pinhas, M., Zetzl, A. K., Barbut, S., \& Marangoni, A. G. (2016). Influence of solvent quality on the mechanical strength of ethylcellulose oleogels. Carbohydrate Polymers, 135, 169-179. http://dx.doi.org/10.1016/j. carbpol.2015.08.050.

Hepworth, P. (2006). In R. J. Farn (Ed.), Non-ionic Surfactants Chemistry and Technology of Surfactants (pp. 133-152). Blackwell Publishing Ltd.

Huang, X., Terech, P., Raghavan, S. R., \& Weiss, R. G. (2005). Kinetics of 5 alpha-cholestan3 beta,6-yl N-(2-naphthyl)carbamate/n-alkane organogel formation and its influence on the fibrillar networks. Journal of the American Chemical Society, 127(12), 4336-4344. http://dx.doi.org/10.1021/Ja0426544.

Kamble, S. R., Udapurkar, P., Nakhat, P. D., Yeole, P. G., \& Biyani, K. R. (2011). Development and evaluation of sorbitan monostearate organogels as a topical delivery system for aceclofenac. Indian Journal of Pharmaceutical Education and Research, 45(1), 65-70.

Laredo, T., Barbut, S., \& Marangoni, A. G. (2011). Molecular interactions of polymer oleogelation. Soft Matter, 7(6), 2734-2743. http://dx.doi.org/10.1039/c0sm00885k.

Lopez-Martinez, A., Charo-Alonso, M. A., Marangoni, A. G., \& Toro-Vazquez, J. F. (2015). Monoglyceride organogels developed in vegetable oil with and without ethyl cellulose. Food Research International, 72, 37-46. http://dx.doi.org/10.1016/j.foodres. 2015.03.019. 
Lopez-Martinez, A., Morales-Rueda, J. A., Dibildox-Alvarado, E., Charo-Alonso, M. A Marangoni, A. G., \& Toro-Vazquez, J. F. (2014). Comparing the crystallization and rheological behavior of organogels developed by pure and commercial monoglycerides in vegetable oil. Food Research International, 64, 946-957. http://dx.doi.org/10.1016/ j.foodres.2014.08.029.

Lupi, F. R., Shakeel, A., Greco, V., Rossi, C. O., Baldino, N., \& Gabriele, D. (2016). A rheological and microstructural characterisation of bigels for cosmetic and pharmaceutical uses. Materials Science and Engineering: C, 69, 358-365.

Martins, A. J., Cerqueira, M. A., Fasolin, L. H., Cunha, R. L., \& Vicente, A. A. (2016). Beeswax organogels: Influence of gelator concentration and oil type in the gelation process. Food Research International, 84, 170-179.

Mezzenga, R., Meyer, C., Servais, C., Romoscanu, A. I., Sagalowicz, L., \& Hayward, R. C. (2005). Shear rheology of lyotropic liquid crystals: A case study. Langmuir, 21, 3322-3333.

Murdan, S., Gregoriadis, G., \& Florence, A. T. (1999). Novel sorbitan monostearate organogels. Journal of Pharmaceutical Sciences, 88(6), 608-614. http://dx.doi.org/10. 1021/Js980342r.

Patel, A. R., Babaahmadi, M., Lesaffer, A., \& Dewettinck, K. (2015). Rheological profiling of organogels prepared at critical gelling concentrations of natural waxes in a triacylglycerol solvent. Journal of Agricultural and Food Chemistry, 63(19), 4862-4869. http://dx.doi.org/10.1021/acs.jafc.5b01548.

Penzes, T., Csoka, I., \& Eros, I. (2004). Rheological analysis of the structural properties effecting the percutaneous absorption and stability in pharmaceutical organogels. Rheologica Acta, 43(5), 457-463. http://dx.doi.org/10.1007/s00397-004-0396-1.

Pernetti, M., van Malssen, K., Kalnin, D., \& Floter, E. (2007). Structuring edible oil with lecithin and sorbitan tri-stearate. Food Hydrocolloids, 21(5-6), 855-861. http://dx.doi. org/10.1016/j.foodhyd.2006.10.023.

Petoukhov, M. V., Franke, D., Shkumatov, A. V., Tria, G., Kikhney, A. G., Gajda, M., .. Svergun, D. I. (2012). New developments in the ATSAS program package for smallangle scattering data analysis. Journal of Applied Crystallography, 45, 342-350. http://dx.doi.org/10.1107/S0021889812007662.

Phan, T. T., Harwell, J. H., \& Sabatini, D. A. (2010). Effects of triglyceride molecular structure on optimum formulation of surfactant-oil-water systems. Journal of Surfactants and Detergents, 13(2), 189-194. http://dx.doi.org/10.1007/s11743-009-1155-1.

Rocha, J. C. B., Lopes, J. D., Mascarenhas, M. C. N., Arellano, D. B., Guerreiro, L. M. R., \& da Cunha, R. L. (2013). Thermal and rheological properties of organogels formed by sugarcane or candelilla wax in soybean oil. Food Research International, 50(1), 318-323. http://dx.doi.org/10.1016/j.foodres.2012.10.043.

Roe, R. -J. (2000). Methods of X-ray and neutron scattering in polymer science. New York: Oxford University Press.

Rogers, M. A., \& Kim, J. H. J. (2011). Rheological assessment of the sol-gel transition for self-assembling low molecular weight gelators. Food Research International, 44(5), 1447-1451. http://dx.doi.org/10.1016/j.foodres.2011.03.014

Rogers, M. A., Pedersen, T., \& Quaroni, L. (2009a). Hydrogen-bonding density of supramolecular self-assembled fibrillar networks probed using synchrotron infrared spectromicroscopy. Crystal Growth \&' Design, 9(8), 3621-3625. http://dx.doi.org/10. 1021/cg900370g.
Rogers, M. A., Wright, A. J., \& Marangoni, A. G. (2009b). Oil organogels: The fat of the future? Soft Matter, 5(8), 1594-1596.

Sahri, M. M., \& Idris, N. A. (2010). Palm stearin as low trans hard stock for margarine. Sain Malaysiana, 39(5), 821-827.

Sanchez, R., Franco, J. M., Delgado, M. A., Valencia, C., \& Gallegos, C. (2011). Rheology of oleogels based on sorbitan and glyceryl monostearates and vegetable oils for lubricating applications. Grasas y Aceites, 62(3), 328-336. http://dx.doi.org/10.3989/gya. 113410.

Sato, K., Yoshimoto, N., Suzuki, M., Kobayashi, M., \& Kaneko, F. (1990). Structure and transformation in polymorphism of petroselinic acid (cis-omega-12-octadecenoic acid). Journal of Physical Chemistry, 94(7), 3180-3185. http://dx.doi.org/10.1021/ J100370a078.

Singh, V. K., Pramanik, K., Ray, S. S., \& Pal, K. (2015). Development and characterization of sorbitan monostearate and sesame oil-based organogels for topical delivery of antimicrobials. AAPS PharmSciTech, 16(2), 293-305. http://dx.doi.org/10.1208/s12249014-0223-7.

Steffe, J. F. (1996). Rheological methods in food process engineering (2nd ed.). East Lansing, MI, USA: Freeman Press.

Terech, P., \& Weiss, R. G. (1997). Low molecular mass gelators of organic liquids and the properties of their gels. Chemical Reviews, 97(8), 3133-3160.

Toro-Vazquez, J. F., Alonzo-Macias, M., Dibildox-Alvarado, E., \& Charo-Alonso, M. A (2009). The effect of tripalmitin crystallization on the thermomechanical properties of candelilla wax organogels. Food Biophysics, 4(3), 199-212. http://dx.doi.org/10. 1007/s11483-009-9118-7.

Toro-Vazquez, J. F., Morales-Rueda, J., Torres-Martinez, A., Charo-Alonso, M. A., Mallia, V. A., \& Weiss, R. G. (2013). Cooling rate effects on the microstructure, solid content, and rheological properties of organogels of amides derived from stearic and (R)-12hydroxystearic acid in vegetable oil. Langmuir, 29(25), 7642-7654. http://dx.doi. org/10.1021/la400809a.

Valoppi, F., Calligaris, S., Barba, L., Šegatin, N., Poklar Ulrih, N., \& Nicoli, M. C. (2017). Influence of oil type on formation, structure, thermal, and physical properties of monoglyceride-based organogel. European Journal of Lipid Science and Technology, 119, 1500549. http://dx.doi.org/10.1002/ejlt.201500549.

Vintiloiu, A., \& Leroux, J. C. (2008). Organogels and their use in drug delivery-A review. Journal of Controlled Release, 125(3), 179-192. http://dx.doi.org/10.1016/j.jconrel 2007.09.014.

Yilmaz, E., \& Ogutcu, M. (2014). Properties and stability of hazelnut oil organogels with beeswax and monoglyceride. Journal of the American Oil Chemists Society, 91(6), 1007-1017. http://dx.doi.org/10.1007/s11746-014-2434-1.

Yu, R., Lin, N., Yub, W., \& Liu, X. Y. (2015). Crystal networks in supramolecular gels: formation kinetics and mesoscopic engineering principles. CrystEngComm, 17 7986-8010.

Zetzl, A., Ollivon, M., \& Marangoni, A. (2009). A coupled differential scanning calorimetry and X-ray study of the mesomorphic phases of monostearin and stearic acid in water. Crystal Growth \& Design, 9(9), 3928-3933. http://dx.doi.org/10. $1021 / \operatorname{cg} 9000285$. 